Review Article

\title{
Biomaterials Design of Culture Substrates for Cell
} Research

\author{
Yasuhiko Tabata*
}

Department of Biomaterials, Field of Tissue Engineering, Institute for Frontier Medical Sciences, Kyoto University, Kyoto, Japan

In general, the body tissue is composed of cells and their local environment. The environment plays an essential role in the attachment, proliferation, migration or differentiation of cells and the subsequent their 3-dimensional organization, which are important biological functions of cells to be investigated for cell-based tissue regeneration. The cell behavior is influenced by the surface and bulk properties of substrates for cell culture, such as the surface hydrophobicity, charge or morphology and the material stiffness. The proliferation and differentiation of cells can be biologically changed by the surface hydrophobicity and charge of culture substrates. The stiffness of culture substrates also modifies the differentiation profiles of stem cells. The combination of culture substrates with bioactive molecules promotes the proliferation and differentiation of stem cells. Various 3-demensional substrates are designed to demonstrate the effect of the mechanical and biological properties on the proliferation and differentiation of cells. Artificial manipulation of cell functions has been experimentally tried. Genetic engineering with transfection technology of plasmid DNA and small interference RNA (siRNA) enabled cells to activate their biological functions and modify their differentiation direction. This paper overviews basic researches on the interaction between cells and cultures substrates with different physicochemical properties. Several cell researches with different culture substrates are introduced to emphasize significance of biomaterial technology in the basic research of stem cells.

Rec./Acc.3/7/2011

${ }^{*}$ Correspondence should be addressed to:

Yasuhiko Tabata, Department of Biomaterials, Field of Tissue Engineering, Institute for Frontier Medical Sciences, Kyoto University, 53 Kawara-cho Shogoin, Sakyo-ku, Kyoto 606-8507, Japan. Phone: +81-75-751-4121, Fax: +81-75-751-4646, E-mail: yasuhiko@frontier.kyoto-u.ac.jp

Key words:

bioactive molecules, biomaterials, cell research, culture substrates, physicochemical properties 


\section{RECENT CHANG IN BIOMATERIALS DEFINITON}

Biomaterials have been known as medical devices, such artificial blood vessels, artificial hearts, bone substitutes, and artificial dialyzer etc, and the surgical devices of sutures, stents, and wound couvers, which clinically contributes to save and improve countless lines of patients. So far, the biomaterial research and development (R\&D) have been directed to design and create the materials which have properties not to induce inflammation reactions and biocompatible to the body tissue. The situation is similar to the R\&D of drug delivery system (DDS) which is one of the important fields in biomaterials. DDS technology has been developed based on the material design to avoid the body responses to the carriers of drugs. Recently, however, the $R \& D$ direction of biomaterials has been being changed to design the materials which are not be recognized by the living system of body, but can oppositely act on the system. The typical example is the biomaterials which can be used for the research field of regenerative medicine. The biomaterials actively manipulate cells to promote their proliferation and differentiation, resulting in enhanced cell-induced tissue regeneration ${ }^{1)}$. Accompanied with the change in the strategy of biomaterials $\mathrm{R} \& \mathrm{D}$, the definition should be changed. The biomaterial is defined as the general name of materials which are used in the body and contacting with biological components of proteins, cells, bacteria, and viruses.

Generally the biomaterials are artificially designed and prepared aiming at the applications to the living system, but their usage is not limited to medical devices and DDS described before. Considering the definition, the substrate used for cell culture is also the biomaterial. The present research of cell biology is being preformed under non-physiological conditions where cells are cultured on the surface of artificial plastic dishes in the artificial solution of neutrients. The conditions are not always alike to those in vivo, and it is practically impossible to exactly investigate the natural features and functions of cells themselves in the body tissue. The basic knowledge of cells obtained from the conventional culture experiments may not reflect the natural functions of cells. Recently it is well recognized that cells generally proliferate and differentiate interacting with the local environment, so-called cell niche $e^{2,3)}$. It is no doubt that the design and creation of cell environment with the property similar to that of the natural niche is quite important to allow to further develop the basic research of cells, which can scientifically improve the cell-based tissue regeneration therapy of next generation. This review briefly introduces the applications of biomaterials and the technology to the research field of cell biology. The conventional and new technologies of culture substrates are overviewed to stimulate readers' interest in the application of biomaterials to basic research in cell biology.

\section{THE CONVENTIONAL RESEARCH OF CELL CULTURE SUBSTRATES}

There are various properties of biomaterials which affect the attachment, proliferation, and differentiation of cells. They can be classified into the surface and bulk properties. The former includes the physicochemical properties of surface hydrophobicity, electric charge, morphology or roughness and the surface modification with the coating and chemical immobilization of cell adhesion molecules. The latter is about the stiffness and intra-structural properties (pore size, distribution, and porosity) of materials.

To make biomaterials biocompatible, several researches have been carried out to investigate the interaction between cells and biomaterials ${ }^{4}$. When cultured on polymer substrates with different surface hydrophobicities, fibroblastic cells attached and proliferated most efficiently for the surface of a certain hydrophobicity range ${ }^{4}$. The positive and negative charges of substrates surface also affected cell behaviors $^{5}$. The cell-material interaction can be explained in terms of the physicochemical theory of adhesion energy in water ${ }^{6}$. Proteins of cell adhesion, such as collagen, fibronectin, and vitronectins, are likely to adsorb onto the surface of optimal hydrophobicity. As the result, the subsequent cell attachment enhanced. A good correlation between the adsorption of the cell adhesive protein and the cell subsequent attachment was observed. The similar tendency of cell-material interaction was observed for macrophages and other cells ${ }^{7,8)}$. This is an universal response of cells to the artificial material, irrespective of the cell type, when it is not necessary to consider biological interaction of ligand-receptor specific binding. Generally, the surface of high hydrophilicity and hydrophobicity suppresses the cell adhesion because of the less protein adsorption onto $\mathrm{it}^{4,9)}$. Based on the results, several experiments have been performed to regulate the cell attachment ${ }^{10-12)}$. 
Surface modification with poly(ethyleneglycol) (PEG) effectively made the substrate surface hydrophilic, resulting in suppressed cell attachment ${ }^{11)}$. This technique is combined with micropatterning technologies. The substrate surface micro-patterned was chemically modified with PEG and/or cell adhesive proteins. The cell attachment and the subsequent proliferation are both regulated by the pattern modified $^{13)}$. The 2-demensional culture substrates with a surface gradient of protein concentration were designed to demonstrate the positive influence of protein concentration on the cell attachment and proliferation $^{14)}$.

Several researches have been reported on the cul- ture substrate of biomaterials surface-modified with various proteins and peptides of cell adhesion ${ }^{15,16)}$. The surface coating and chemical immobilization with collagen, fibronectin, and Arg-Gly- Asp-Ser (RGDS) peptides enhanced the initial attachment of cells onto the substrates and the subsequent proliferation ${ }^{17)}$. Similar to the normal fibroblastic cells, the proliferation of adult stem cells, such as bone marrow-derived mesenchymal stem cells (MSC) was also promoted ${ }^{18)}$. The surface hydrophobicity and electric charge also affected the proliferation and differentiation of bone marrow- and fat-derived $\mathrm{MSC}^{19)}$. Their pattern of bone and fat differentiation was modified by the surface properties ${ }^{20)}$.
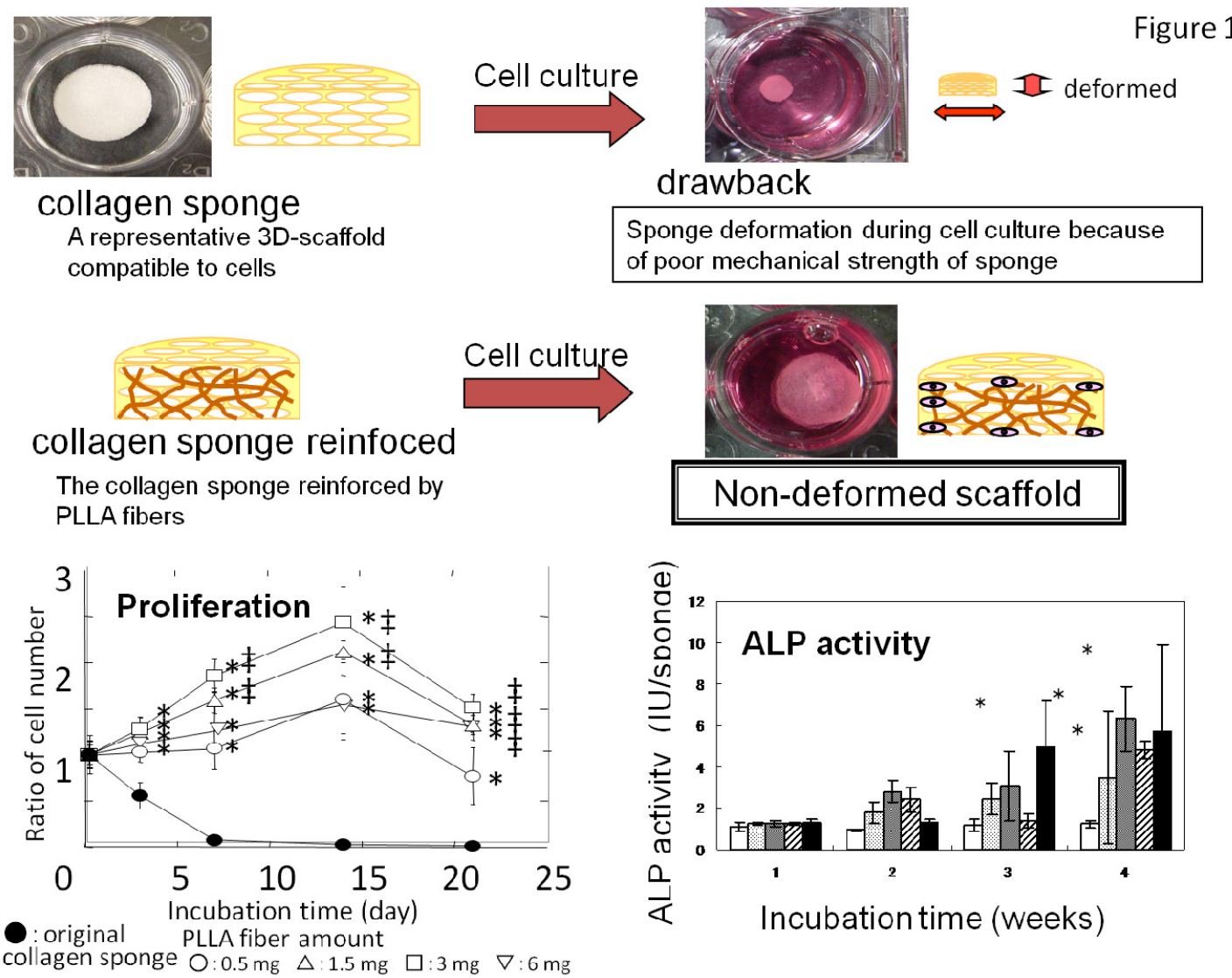

Fig. 1

Enhanced proliferation and osteogenic differentiation of rat MSC in fiber-reinforced collagen sponge scaffold. The incorporation of poly(L-lactic acid) (PLLA) fibers enabled the sponge to increase the mechanical strength. The mechanical reinforcement suppressed the sponge deformation during the cell culture, resulting in the maintenance of intra-space for cell proliferation and differentiation.

As the 3-demensinal (3D) substrates of cell culture, polymer sponges and non-woven fabrics or porous ceramics have been prepared to evaluate their feasibility in culture substrate ${ }^{21)}$. There are some re- search reports where an enhanced cell proliferation was observed for the 3D culture substrate comparing with for the $2 \mathrm{D}$ one ${ }^{22}$. However, the results are still controversial because little comparison study has 
been reported for different dimensional substrates prepared from the same material.

There are several points to be improved for the substrate design. The first point is to improve the mechanical property of substrates. For example, the 3D sponge of collagen is too mechanically soft to maintain their pore structure and interconnectivity. Since during the cell culture, the sponge is often shrunken and deformed, cells do not proliferate in the sponge and sometimes died. This is because there is no space for cell proliferation and no supply of oxygen and nutrients to cells. As one trial to tackle the issue, fibers and ceramic granules were incorporated into the 3D substrate of sponge type to mechanically reinforce it without any loss of the inherent cell compatibility of sponge material ${ }^{23)}$. Rat MSC were proliferated and differentiated into bone cells in the reinforced sponge substrate to a significant great extent compared with the original sponge $^{23,24)}$. (Fig.1)

\section{NEW DIRECTION OF RESEACH FOR CELL CULTRUE SUBSTRATES}

In addition to the surface hydrophobicity and electric charge, the surface morphology is also an important factor to affect the cell behaviors ${ }^{13,25)}$. Recently nanotechnology has been developed and enabled the surface fabrication of materials in a nano-ordered size ${ }^{26}$. The nano-ordered surface roughness was found to affect the proliferation of cells ${ }^{25)}$. In addition, electro-spin technologies can produce the non-woven fabrics with nano-ordered fibers. The non-woven fabrics electrospun have been investigated for the culture substrates. It is demonstrated that the fiber diameter and density as well as the property of fiber materials modified the pattern of cell attachment and proliferation as well as the subsequent cell differentiation ${ }^{27}$.

Figure 2

\section{The pattern of osteogenic differentiation of rat MSC in an alginate sponges with a concentration gradient of bFGF was in good accordance with that of bFGF concentration.}
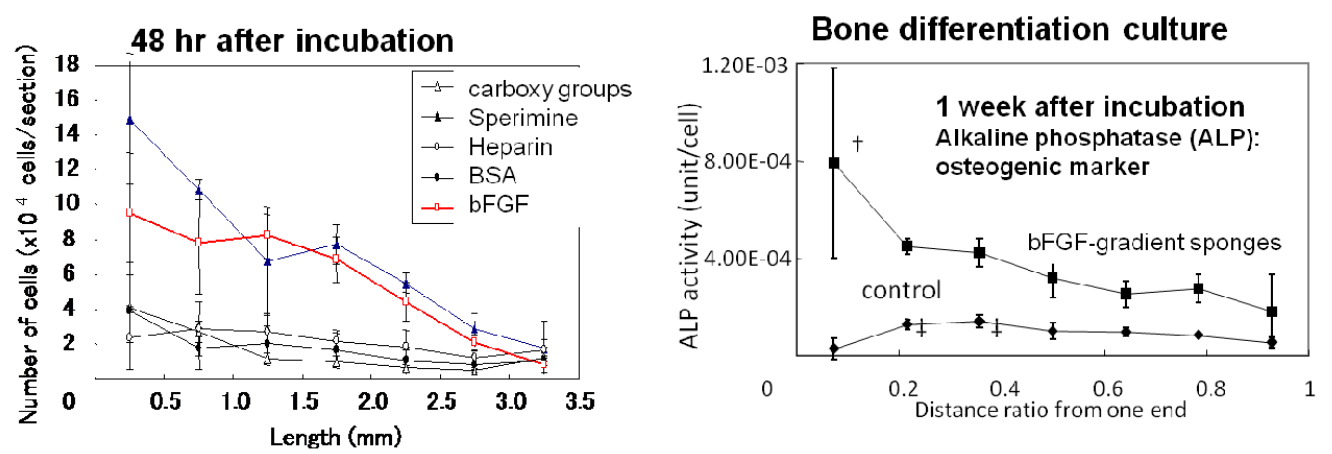

Fig. 2

Osteogenic differentiation of rat MSC in an alginate sponge with a concentration gradient of bFGF. The cell differentiation depended on the amount of bFGF immobilized.

The substrate stiffness is one of the factors contributing to the patterns of cell attachment, proliferation, and differentiation ${ }^{28)}$. It is reported that stem cells are differentiated into different types of cells in the response to the stiffness of culture substrates ${ }^{28}$. For example, when cultured on a substrate with the stiffness close to that of fat tissue, the cells tended to differentiate into fat cells. The type of cells to be differentiated depended on the stiffness of culture substrates. In addition, the surface topology of shape and area capable for cell attachment also modified the cell proliferation and differentiation ${ }^{13)}$. The cell attachment and the shape of cells attached were greatly influenced by the surface topography of culture substrates ${ }^{29,30)}$. This series of research results strongly indicate that the property of cell substrates 
greatly affects the fate of cells cultured.

The technology to immobilize protein onto the surface of culture substrates has been improved recently. Distinct from the simple coating and immobilization, the immobilization of bioactive proteins in a well-oriented fashion has been tried to demonstrate the better property to promote the proliferation of stem cells than the conventional random-immobilization system $^{31-34)}$. Various technologies for the well-oriented protein immobilization on the substrate surface are reported by use of histidine-tagged protein $^{34)}$, antibody Fc-hybrid protein ${ }^{33)}$, and DNA hybridization $^{32)}$. It is reported that a fraction of hematopoietic stem cells was efficiently proliferated on the man-made substrate of biomaterials surface-modified in the well-oriented fashion ${ }^{35)}$.

It is well recognized that cells generally interact with their surrounding environment to survive and biologically function or allow to determine their direction of differentiation ${ }^{36,37)}$. It is found in the developmental biology that the tissue organization in the fertilized egg is determined by the change of biological materials in the local environment around cells devided ${ }^{36,37)}$. The concentration and steric position of proteins and active molecules in the local environment of cells must be a biological cue to determine the fate of cells. 3D culture substrates with a concentration gradient of bioactive protein are designed and prepared ${ }^{38,39)}$. The concentration gradient could regulate the proliferation and differentiation of cells in the 3D sponge substrate ${ }^{38)}$. (Fig. 2)

DDS technology and methodology play an important role in the basic research of stem cells biology. Bio-signaling molecules to regulate the functions and fate of stem cells have been identified and available to use. Generally the molecules are biologically instable. To expect their biological activities, the combination with DDS technology is practically necessary. This necessity is not only for the in vivo therapeutic usage of in-stable molecules, but also for their research applications. The DDS technology is undoubtedly useful to develop the basic research of stem cells and consequently realize cell-based regeneration therapy. In addition to the growth factors of bio-signaling protein, gene is an important research tool for cell biology. Gene transfection has been extensively used as a standard research procedure. One of the keys for gene transfection is to enhance the transfection efficiency. Generally, the transfection efficiency of viral vectors is higher than that of non-viral carriers. In addition, the time period of gene expression is short for the non-viral carriers. However, from the viewpoint of easiness to use, the non-viral carrier is experimentally preferable. Therefore, several trials combining with the DDS technology has been performed to improve the in vivo and in vitro efficiency of gene transfection. The gene carriers of biomaterials, such as cationic liposomes and polymers, have been actively investigated to enhance the level of gene expression ${ }^{40}$. However, little has been investigated on the research trials to prolong the time period of gene expression. For example, the release of plasmid DNA from a biodegradable hydrogel of cationized gelatin derivatives enhanced the level of gene expression as well as prolonged the time period of expression ${ }^{41,42)}$. The time period of gene expression could be regulated by changing that of prasmid DNA release.

This technology of gene delivery is applicable to genetically engineer cells for their functional activation, which is useful to develop the basic science of stem cells biology and enhance the therapeutic efficiency of cell transplantation. There are some cases where the transplantation of stem cells alone does not always induce a therapeutic effect clinically acceptable. A promising and practical way to break through this problem would be to genetically engineer stem cells and activate their biological functions. So far, such cell activation has been tried by using virus vectors ${ }^{43,44)}$. This trial has been of great success, but the good results with virus vectors cannot be easily applied to clinical therapies. Some trials have been reported on the genetic engineering of stem cells by non-viral carriers ${ }^{45)}$. The intracellular release of plasmid DNA with biodegradable hydrogel microspheres could genetically engineer endothelial progenitor cells (EPC) to activate their angiogenic function. When injected into an animal model of cardiac infarction, the EPC genetically engineered showed superior therapeutic efficacy to the original $\mathrm{EPC}^{46)}$. In addition to the microspheres for the intracellular release of plasmid DNA, a new non-viral carrier has been prepared from cationized polysaccharides. The carrier of gene transfection enabled plasmid DNA to enhance the level of gene expression for stem cells with less cytotoxicity than cationized liposomes commercially available. The gene transfection with the cationized polysaccharide was effective in genetically engineering the biological function of stem cells ${ }^{40}$. The level and time period of gene expression by the non-viral carrier was further improved by devising the methodology of gene 
transfection culture, such as the reverse transfection method and the bioreactor combination ${ }^{47)}$. The reverse transfection technology was effective in gene transfection for monkey MSC to allow them to differentiate into nerve cells without any cytotoxicity ${ }^{48)}$. The MSC differentiated functioned well to show good therapeutic efficacy for nerve degenerative disease ${ }^{49)}$. Thus, the gene engineering technology with biomaterials is an effective and powerful research tool to develop the basic research of stem cells biology and medicine. The DDS technology of gene transfection will be allow bioactive substances other than genes to internalize into cells to investigate their biological functions in stem cells biology. The combination of small interfering RNA (siRNA) with the cationized polysaccharide of non-viral carrier genetically regulated the function of stem cells and modified the direction of cell differentiation ${ }^{50)}$. The biomaterial carriers to internalize bioactive substances into cells can artificially modify cell functions, which is useful to elucidate the mechanism of cell proliferation and differentiation and regulate the cell behavior based on the results obtained. (Fig. 3)

\section{Gene transfection of monkey stem cells was achieved.} Differentiation of bone marrow-derived mesenchymal stem
cells to dopaminagic neural cells
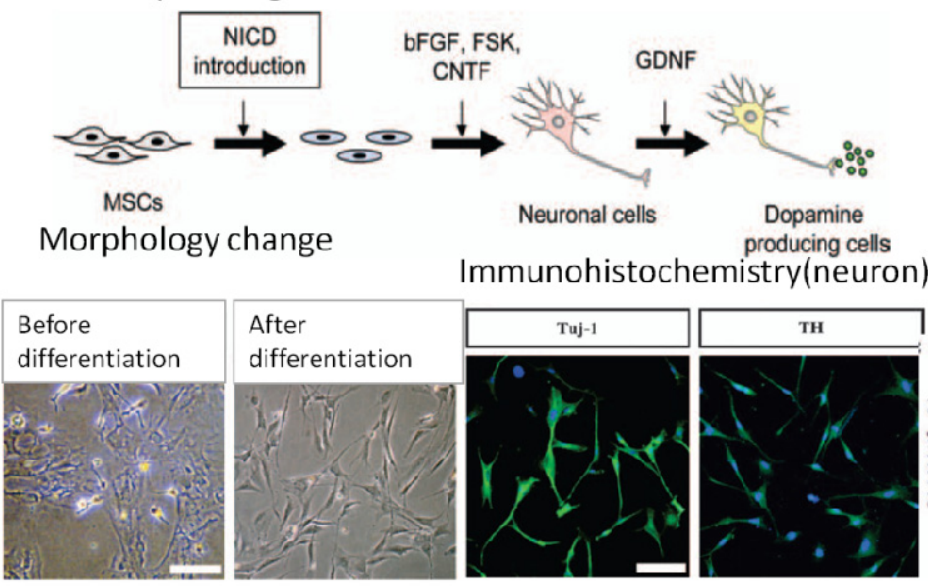

\section{Less cytotoxicity}

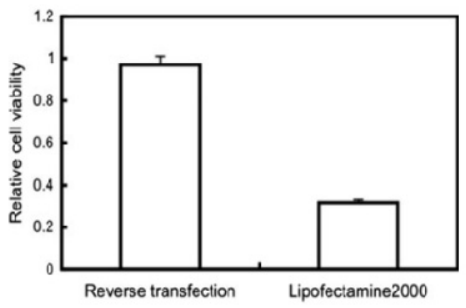

Dopamine production
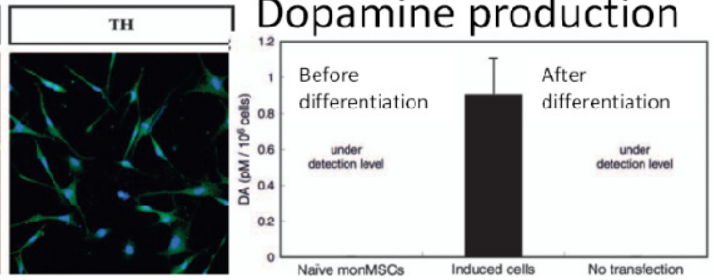

Fig. 3

Neural differentiation of monkey MSC by the reverse transfection technology with cationic polysaccharide. The MSC were differentiated into Dopamine-secreting neuron cells without cell death.

\section{CLOSING REMARKS}

The tissue regeneration therapy - a new therapy based on the healing potential of patients themselves to induce tissue regeneration through cells transplantation and tissue engineering with biomaterials is the third therapy following the reconstructive surgery and organ transplantation. To achieve the regeneration therapy by use of tissue engineering technologies, substantial collaborative research between biomaterial, pharmaceutical, biological, and clinical scientists is needed. Even if superior stem cells can be practically obtained, it is impossible to therapeutically treat patients only by transplanting the cells prepared even combining with the scientific knowledge of cells and the related substances, unless a local environment of cells suitable to promote the proliferation and differentiation is created and provided properly. To create the cell environment, it is no doubt that further development of stem cell researches is needed. Biomaterials and the technology will experimentally assist the research development.

However, one of the main problems to create the regeneration environment at present is the absolute 
shortage of biomaterial researchers who investigate the cell scaffold, the DDS, and cell culture, aiming at tissue regeneration and the biological substitution of organ functions. Such researchers must have knowledge in medicine, dentistry, biology and pharmacology, in addition to material sciences. It is indispensable to educate the researchers of an interdisciplinary field who have an engineering background and can also understand basic biology, medicine and clinical medicine.

Biomaterials are not only for materials to design and prepare medical devices and DDS for drug therapy, but also substantially important and necessary to further develop the basic research of stem cell biology. However, such the concept of biomaterial applications is still in its infancy, although biologists have recently recognized that the materials currently available are not always enough to proceed the research of cell biology and deeply investigate the cellular mechanism. The increasing significance of biomaterials to design the culture substrate for the local environment of cells in future will help progress in the basic research of cell biology which scientifically supports the tissue regeneration therapy of next generation. We will be happy if this review stimulates readers' interest in the idea and research field of biomaterials to assist understanding how important biomaterials are to develop the basic research of stem cells biology and medicine as well as realize tissue regeneration therapy.

\section{REFERENCES}

1) Tabata Y: Biomaterial technology for tissue engineering applications. J R Soc Interface. 2009; 6 Suppl 3: S311-324.

2) Wilson A, Trumpp A: Bone-marrow haematopoietic-stem-cell niches. Nat Rev Immunol. 2006; 6: 93-106.

3) Morrison SJ, Spradling AC: Stem cells and niches: mechanisms that promote stem cell maintenance throughout life. Cell. 2008; 132: 598-611.

4) Tamada Y, Ikada Y: Fibroblast growth on polymer surfaces and biosynthesis of collagen. J Biomed Mater Res. 1994; 28: 783-789.

5) Schneider GB, English A, Abraham M, Zaharias R, Stanford C, Keller J: The effect of hydrogel charge density on cell attachment. Biomaterials. 2004; 25 : 3023-3028.

6) Y. Ikada MS, and Y. Tamada: Polymers as Bioma- terials. SM Shalaby, AS Hoffman, BD Ratner, and TA Horbett Ed, Plenum Press. 1985; 135.

7) Tabata Y, Ikada Y: Effect of the size and surface charge of polymer microspheres on their phagocytosis by macrophage. Biomaterials. 1988; 9: 356-362.

8) Altankov G, Grinnell F, Groth T: Studies on the biocompatibility of materials: fibroblast reorganization of substratum-bound fibronectin on surfaces varying in wettability. J Biomed Mater Res. 1996; 30: $385-391$.

9) $\mathrm{Du} \mathrm{H}$, Chandaroy P, Hui SW: Grafted poly-(ethylene glycol) on lipid surfaces inhibits protein adsorption and cell adhesion. Biochim Biophys Acta. 1997; 1326: 236-248.

10) Kumashiro $Y$, Yamato $M$, Okano T: Cell attachment-detachment control on temperature-responsive thin surfaces for novel tissue engineering. Ann Biomed Eng. 2010; 38: 1977-1988.

11) Tessmar JK, Gopferich AM: Customized PEG-derived copolymers for tissue-engineering applications. Macromol Biosci. 2007; 7: 23-39.

12) Ikada Y: Challenges in tissue engineering. J R Soc Interface. 2006; 3: 589-601.

13) Bettinger CJ, Langer R, Borenstein JT. Engineering substrate topography at the micro- and nanoscale to control cell function. Angew Chem Int Ed Engl. 2009; 48: 5406-5415.

14) Keenan TM, Folch A: Biomolecular gradients in cell culture systems. Lab Chip. 2008; 8: 34-57.

15) Dellatore SM, Garcia AS, Miller WM: Mimicking stem cell niches to increase stem cell expansion. Curr Opin Biotechnol. 2008; 19: 534-540.

16) Mrksich M: Using self-assembled monolayers to model the extracellular matrix. Acta Biomater. 2009; 5: 832-841.

17) Kobayashi H, Ikada Y: Covalent immobilization of proteins on to the surface of poly(vinyl alcohol) hydrogel. Biomaterials. 1991; 12: 747-751.

18) Rehfeldt F, Engler AJ, Eckhardt A, Ahmed F, Discher DE: Cell responses to the mechanochemical microenvironment--implications for regenerative medicine and drug delivery. Adv Drug Deliv Rev. 2007; 59: 1329-1339.

19) Curran JM, Chen R, Hunt JA: The guidance of human mesenchymal stem cell differentiation in vitro 
by controlled modifications to the cell substrate. Biomaterials. 2006; 27: 4783-4793.

20) Inoue S, Iida $Y$, Otani $Y$, Hirano $Y$, Tabata $Y$ : Adhesion behavior of human adipo-stromal cells on self-assembled monolayers with different surface densities or gradients of RGD peptide. J Biomater Sci Polym Ed. 2009; 20: 495-510.

21) Auxenfans C, Builles N, Andre V, Lequeux C, Fievet $\mathrm{A}$, Rose $\mathrm{S}$, et al.: [Porous matrix and primary-cell culture: a shared concept for skin and cornea tissue engineering]. Pathol Biol (Paris). 2009; 57: $290-298$.

22) Inoue $S$, Imamura $M$, Hirano $Y$, Tabata $Y$ : Adhesion and proliferation of human adipo-stromal cells for two- or three-dimensional poly(ethylene terephthalate) substrates with or without RGD immobilization. J Biomater Sci Polym Ed. 2009; 20: 721-736.

23) Hiraoka Y, Kimura Y, Ueda H, Tabata Y: Fabrication and biocompatibility of collagen sponge reinforced with poly(glycolic acid) fiber. Tissue Eng. 2003; 9: 1101-1112.

24) Takahashi $Y$, Yamamoto $M$, Tabata $Y$ : Osteogenic differentiation of mesenchymal stem cells in biodegradable sponges composed of gelatin and beta-tricalcium phosphate. Biomaterials 2005; 26: 3587-3596.

25) Ito Y: Surface micropatterning to regulate cell functions. Biomaterials. 1999; 20: 2333-2342.

26) Gates BD, Xu Q, Stewart M, Ryan D, Willson CG, Whitesides GM: New approaches to nanofabrication: molding, printing, and other techniques. Chem Rev. 2005; 105: 1171-1196.

27) Nisbet DR, Forsythe JS, Shen W, Finkelstein DI, Horne MK: Review paper: a review of the cellular response on electrospun nanofibers for tissue engineering. J Biomater Appl. 2009; 24: 7-29.

28) Engler AJ, Sen S, Sweeney HL, Discher DE: Matrix elasticity directs stem cell lineage specification. Cell. 2006; 126: 677-689.

29) Kononen M, Hormia M, Kivilahti J, Hautaniemi J, Thesleff I: Effect of surface processing on the attachment, orientation, and proliferation of human gingival fibroblasts on titanium. J Biomed Mater Res. 1992; 26: 1325-1341.

30) Degasne I, Basle MF, Demais V, Hure G, Lesourd
M, Grolleau B, et al.: Effects of roughness, fibronectin and vitronectin on attachment, spreading, and proliferation of human osteoblast-like cells (Saos-2) on titanium surfaces. Calcif Tissue Int. 1999; 64: 499-507.

31) Iuliano DJ, Saavedra SS, Truskey GA: Effect of the conformation and orientation of adsorbed fibronectin on endothelial cell spreading and the strength of adhesion. J Biomed Mater Res. 1993; 27: 1103-1113.

32) Boozer C, Ladd J, Chen S, Jiang S: DNA-directed protein immobilization for simultaneous detection of multiple analytes by surface plasmon resonance biosensor. Anal Chem. 2006; 78: 1515-1519.

33) Aoki T, Takahashi Y, Koch KS, Leffert HL, Watabe $\mathrm{H}$ : Construction of a fusion protein between protein $\mathrm{A}$ and green fluorescent protein and its application to western blotting. FEBS Lett. 1996; 384: 193-197.

34) Nakaji-Hirabayashi $T$, Kato $K$, Arima $Y$, Iwata $H$ : Oriented immobilization of epidermal growth factor onto culture substrates for the selective expansion of neural stem cells. Biomaterials. 2007; 28: $3517-$ 3529 .

35) Kertesz Z, Vas V, Kiss J, Urban VS, Pozsonyi E, Kozma A, et al.: In vitro expansion of long-term repopulating hematopoietic stem cells in the presence of immobilized Jagged-1 and early acting cytokines. Cell Biol Int. 2006; 30: 401-405.

36) Song $\mathrm{X}$, Zhu $\mathrm{CH}$, Doan $\mathrm{C}$, Xie T: Germline stem cells anchored by adherens junctions in the Drosophila ovary niches. Science. 2002; 296: 1855-1857.

37) Li L, Xie T: Stem cell niche: structure and function. Annu Rev Cell Dev Biol. 2005; 21: 605-631.

38) Griffith LG, Swartz MA: Capturing complex 3D tissue physiology in vitro. Nat Rev Mol Cell Biol. 2006; 7: 211-224.

39) Yamamoto M, Yanase K, Tabata Y: Generation of Type I Collagen Gradient in Polyacrylamide Hydrogels by a Simple Diffusion-Controlled Hydrolysis of Amide Groups. Materials. 2010; 3: 2393-2404.

40) Jo JI, Tabata Y: Non-viral gene transfection technologies for genetic engineering of stem cells. Eur J Pharm Biopharm. 2008; 68: 90-104.

41) Kushibiki T, Tabata Y: A new gene delivery system based on controlled release technology. Current drug delivery. 2004; 1: 153-163. 
42) Kushibiki T, Tomoshige R, Fukunaka Y, Kakemi M, Tabata Y: In vivo release and gene expression of plasmid DNA by hydrogels of gelatin with different cationization extents. J Control Release. 2003; 90: 207-216.

43) Tambara K, Premaratne GU, Sakaguchi G, Kanemitsu N, Lin X, Nakajima H, et al.: Administration of control-released hepatocyte growth factor enhances the efficacy of skeletal myoblast transplantation in rat infarcted hearts by greatly increasing both quantity and quality of the graft. Circulation. 2005; 112: I129-I134.

44) Soto-Gutierrez A, Kobayashi N, Rivas-Carrillo JD, Navarro-Alvarez N, Zhao D, Okitsu T, et al.: Reversal of mouse hepatic failure using an implanted liver-assist device containing ES cell-derived hepatocytes. Nat Biotechnol. 2006; 24: 1412-1419.

45) Yamamoto M, Tabata Y: Tissue engineering by modulated gene delivery. Adv Drug Deliv Rev. 2006; 58: 535-554.

46) Nagaya N, Kangawa K, Kanda M, Uematsu M, Horio T, Fukuyama N, et al.: Hybrid cell-gene therapy for pulmonary hypertension based on phagocytosing action of endothelial progenitor cells. Circulation. 2003; 108: 889-895.

47) Okazaki A, Jo J, Tabata Y: A reverse transfection technology to genetically engineer adult stem cells. Tissue Engineering. 2007; 13: 245-251.

48) Nagane K, Kitada M, Wakao S, Dezawa M, Tabata $\mathrm{Y}$ : Practical induction system for dopamine-producing cells from bone marrow stromal cells using spermine-pullulan-mediated reverse transfection method. Tissue Eng Part A. 2009; 15: 1655-1665.

49) Wakao S, Hayashi T, Kitada M, Kohama M, Matsue $\mathrm{D}$, Teramoto $\mathrm{N}$, et al.: Long-term observation of auto-cell transplantation in non-human primate reveals safety and efficiency of bone marrow stromal cell-derived Schwann cells in peripheral nerve regeneration. Exp Neurol. 2010; 223: 537-547.

50) Nagane K, Jo J, Tabata Y: Promoted adipogenesis of rat mesenchymal stem cells by transfection of small interfering RNA complexed with a cationized dextran. Tissue Eng Part A. 2010; 16: 21-31. 